\title{
Nonextensivity and the $q$-distribution of a relativistic gas under an external electromagnetic field
}

\author{
LIU ZhiPeng ${ }^{1,2}$, GUO LiNa ${ }^{1} \&$ DU JiuLin ${ }^{1 *}$ \\ ${ }^{1}$ Department of Physics, School of Science, Tianjin University, Tianjin 300072, China; \\ ${ }^{2}$ Department of Fundamental Subject, Tianjin Institute of Urban Construction, Tianjin 300384, China
}

Received April 6, 2011; accepted May 18, 2011

\begin{abstract}
We investigate the nonextensivity and the $q$-distribution of a relativistic gas under an external electromagnetic field. We derive an expression for the nonextensive parameter $q$ based on the relativistic generalized Boltzmann equation, the relativistic $q-H$ theorem and the relativistic version of the $q$-power-law distribution function in the nonextensive $q$-kinetic theory. We thus provide the connection between the parameter $q \neq 1$ and the spatial-temporal derivatives of the temperature field of the gas as well as the four-potential, and thereby present a clearly physical meaning for the nonextensivity for the relativistic gas.
\end{abstract}

nonextensivity, $q$-distribution, relativistic gas

Citation: $\quad$ Liu Z P, Guo L N, Du J L. Nonextensivity and the $q$-distribution of a relativistic gas under an external electromagnetic field. Chinese Sci Bull, 2011, 56: 3689-3692, doi: 10.1007/s11434-011-4750-2

In recent years, nonextensive statistical mechanics (NSM) has been considered as one of the generalizations of Boltzmann-Gibbs (BG) statistics. NSM has been studied on the basis of Tsallis entropy [1], with the nonextensive parameter $q$ different from unity, in the form of

$$
S_{q}=\frac{k}{1-q}\left(\sum_{i=1}^{W} p_{i}^{q}-1\right)
$$

where $p_{i}$ is the probability that the system under consideration is in its $i$ th configuration and $k$ is Boltzmann constant. This entropy is nonextensive for the parameter $q \neq 1$ and the deviation of $q$ from unity is said to represent the degree of nonextensivity. Taking the limit $q \rightarrow 1, S_{q}$ reduces to the BG entropy, $S=k \Sigma_{i} p_{i} \ln p_{i}$, and extensivity is recovered.

NSM has attracted a great deal of attention both from theoretical study and experimental observation [2]. When many traditional theories in BG statistics have been generalized in the framework of NSM and have been applied to various interesting fields, we need to know under what

*Corresponding author (email: jldu@tju.edu.cn) circumstances, e.g. which class of nonextensive systems and under what physical situations, can NSM be used for the statistical description. Thus, understanding the physical meaning of $q$ and determining this parameter from the underlying microscopic dynamics of the systems under consideration has become very important problems in NSM and for its applications. In this aspect, some theoretical research has been done (e.g. see [3-11] and the references therein), in which self-gravitating systems and plasma systems have offered the best framework to research the nonextensive effects [4-8,12-24].

In particular, the expression for the nonextensive parameter $q$ has been determined rigorously by the generalized Maxwell-Boltzmann (MB) distribution, the generalized Boltzmann equation and the $q-H$ theorem in the framework of NSM, thus the nonextensivity is related to some nonequilibrium properties of the systems with long-range interactions (e.g. gravitational forces and Coulombian forces) [4-11]. In other words, we now know that, for NSM, the parameter $q$ different from unit is related to the temperature gradient and the potentials of the systems such as self-gravitating systems and plasma systems. Thus NSM can be 
reasonably applied to describing the thermodynamic properties of the systems under an external field when they are in the nonequilibrium stationary-state. Recently, the above theory has led to an experimental test of NSM using the solar sound speeds in the helioseismological measurements [21].

Recently, the stationarity, ergodicity and entropy in relativistic systems were analyzed [25], and a close connection between time parameters and entropy in special relativity was revealed. The generalized Boltzmann equation and the $q-H$ theorem for a relativistic case are studied in the $q$-kinetic theory, where an expression of the nonextensive four-entropy flux is given [26] by

$$
S_{q}^{\mu}=-k_{\mathrm{B}} c^{2} \int \frac{\mathrm{d}^{3} p}{E} p^{\mu} f^{q} \ln _{q} f .
$$

Following the lines from the kinetic theory presented in $[4,5,8]$, in this work, we derive the expression of the nonextensive parameter $q \neq 1$ for the relativistic gas and then present it a physical interpretation for a relativistic gas under an external electromagnetic field.

\section{The physical interpretation for $q$-parameter}

We consider a relativistic gas of $\mathrm{N}$-point particles of mass $m$ enclosed in a volume $V$ and under the action of an external Lorentz four-force field,

$$
F^{\mu}(x, p)=-\frac{Q}{m c} F^{\mu v}(x) p_{v},
$$

where the particles have four-momentum $p \equiv p^{\mu}=(E / c, \boldsymbol{p})$ at the point $x \equiv x^{\mu}=(c t, \boldsymbol{r})$ of the space-time, with the energy $E / c=\sqrt{\boldsymbol{p}^{2}+m^{2} c^{2}}, Q$ is the charge of the particle, and $F^{u v}$ is the Maxwell electromagnetic tensor. The index $\mu$ takes its usual values $0,1,2,3$, which identify one time and three space coordinates. The states of this gas can be characterized by a Lorentz invariant one-particle distribution function, $f_{q}(x, p)$. Thus at each time $t, f_{q}(x, p) \mathrm{d}^{3} x \mathrm{~d}^{3} p$ gives the number of particles in the volume element $\mathrm{d}^{3} x \mathrm{~d}^{3} p$ around the space position $\boldsymbol{x}$ and momentum $\boldsymbol{p}$. The evolution equation of the relativistic distribution function is assumed to be the relativistic generalized Boltzmann equation in the $q$ kinetic theory [26]:

$$
p^{\mu} \partial_{\mu} f_{q}+m F^{\mu} \frac{\partial f_{q}}{\partial p^{\mu}}=C_{q}\left(f_{q}\right),
$$

where $\partial_{\mu}=\left(c^{-1} \partial t, \nabla\right)$ are the respective derivatives with respect to the time-space coordinates, and $C_{q}(f)$ is the relativistic $q$-collisional term. The relativistic version of the power-law distribution in the framework of NSM can be obtained as a natural consequence of the relativistic $q-H$ theorem:

$$
f_{q}(x, p)=\left\{1-(1-q)\left[\alpha(x)+\beta_{\mu} p^{\mu}\right]\right\}^{1 / 1-q},
$$

where $\alpha(x)$ and $\beta_{\mu}(x)$ are arbitrary space-time-dependent parameters. For the above relativistic gas in the presence of an external electromagnetic field, it can be written $[26,27]$ as

$$
f_{q}(x, p)=n B_{q}\left[1-(1-q)\left(\frac{u-\left[p^{\mu}+c^{-1} Q A^{\mu}(x)\right] U_{\mu}}{k T}\right)\right]^{\frac{1}{1-q}},
$$

where $n$ is the particle number density, $B_{q}$ a normalization constant, $u$ the Gibbs function per particles, $U_{\mu}$ the mean four-velocity of the gas and $A^{\mu}(x)$ is the four-potential. It is clear that, in the limit $q \rightarrow 1$, eq. (6) recovers to the wellknown relativistic Juttner distribution [28,29]:

$$
f(x, p)=n B \exp \left(\frac{u-\left[p^{\mu}+c^{-1} Q A^{\mu}(x)\right] U_{\mu}}{k T}\right) .
$$

To determine the expression for the nonextensive parameter, according to the relativistic $q-H$ theorem, the solution of eq. (4) will evolve towards the power-law distribution function, eq. (6), the $q$-collision term vanishes, $C_{q}\left(f_{q}\right)=0$, and eq. (4) reduces to

$$
p^{\mu} \partial_{\mu} f_{q}+m F^{\mu} \frac{\partial f_{q}}{\partial p^{\mu}}=0 .
$$

In other words [30], the distribution function (6) must satisfy eq. (8). For the sake of convenience, we can write the equation for $f_{q}^{1-q}(x, p)$ as

$$
p^{\mu} \partial_{\mu} f_{q}^{1-q}+m F^{\mu} \frac{\partial f_{q}^{1-q}}{\partial p^{\mu}}=0 .
$$

From eq. (6), we have

$f^{1-q}(x, p)=n^{1-q} B_{q}^{1-q}\left[1-(1-q)\left(\frac{\mu-\left[p^{\mu}+c^{-1} Q A^{\mu}(x)\right] U_{\mu}}{k_{\mathrm{B}} T}\right)\right]$.

Then,

$$
\begin{aligned}
\partial_{\mu} f^{1-q}(x, p)= & {\left[1-(1-q)\left(\frac{\mu-\left[p^{\mu}+c^{-1} Q A^{\mu}\right] U_{\mu}}{k_{\mathrm{B}} T}\right)\right] } \\
& \times \partial_{\mu}\left(n B_{q}\right)^{1-q}-\left[\left(n B_{q}\right)^{1-q}\right]\left[\frac{-u}{k_{\mathrm{B}} T^{2}} \partial_{\mu} T\right. \\
& +\frac{p^{\mu} U_{\mu}}{k_{\mathrm{B}} T^{2}} \partial_{\mu} T+\frac{c^{-1} Q A^{\mu}(x) U_{\mu}}{k_{\mathrm{B}} T^{2}} \partial_{\mu} T \\
& \left.-\frac{c^{-1} Q \partial_{\mu} A^{\mu}(x)}{k_{\mathrm{B}} T} U_{\mu}\right]
\end{aligned}
$$


and

$$
\frac{\partial f^{1-q}}{\partial p^{\mu}}=\left(n B_{q}\right)^{1-q} \frac{U_{\mu}}{k_{\mathrm{B}} T} .
$$

Substituting eqs. (11) and (12) into eq. (9), we have

$$
\begin{aligned}
& p^{\mu} \partial_{\mu}\left(n B_{q}\right)^{1-q}-p^{\mu}(1-q) \frac{u}{k_{\mathrm{B}} T} \partial_{\mu}\left(n B_{q}\right)^{1-q} \\
& +\frac{\left[p^{\mu}+c^{-1} Q A^{\mu}(x)\right] U_{\mu}}{k_{\mathrm{B}} T} \partial_{\mu}\left(n B_{q}\right)^{1-q} \\
& -p^{\mu}\left(n B_{q}\right)^{1-q}\left[\frac{-u+p^{\mu} U_{\mu}+c^{-1} Q A^{\mu}(x) U_{\mu}}{k_{\mathrm{B}} T^{2}} \partial_{\mu} T\right. \\
& \left.-\frac{c^{-1} Q \partial_{\mu} A^{\mu}(x)}{k_{\mathrm{B}} T} U_{\mu}\right] \\
& -\frac{Q}{c} F^{\mu v} p_{v}\left(n B_{q}\right)^{1-q}(1-q) \frac{U_{\mu}}{k_{\mathrm{B}} T}=0 .
\end{aligned}
$$

Eq. (13) is considered identically null for any arbitrary variable $p$, and hence the sum of the coefficients of each power for $p$ must be zero. We therefore derive the sum of the coefficients in eq. (13), for the first-order term in $p$, as

$$
\begin{aligned}
& p^{\mu} \partial_{\mu}\left(n B_{q}\right)^{1-q}-p^{\mu}(1-q) \frac{u}{k_{\mathrm{B}} T} \partial_{\mu}\left(n B_{q}\right)^{1-q} \\
& +p^{\mu}(1-q) \frac{c^{-1} Q A^{v}(x) U^{\mu}}{k_{\mathrm{B}} T} \partial_{\mu}\left(n B_{q}\right)^{1-q} \\
& -p^{\mu}\left(n B_{q}\right)^{1-q}(1-q)\left[\frac { 1 } { k _ { \mathrm { B } } T ^ { 2 } } \left(-u+c^{-1} Q A^{\mu}(x) U_{\mu} \partial_{\mu} T\right.\right. \\
& \left.-\frac{c^{-1} Q A^{\mu}(x)}{k_{\mathrm{B}} T} U_{\mu}\right]-\frac{Q}{c} F^{\mu v} p_{v}\left(n B_{q}\right)^{1-q}(1-q) \frac{U_{v}}{k_{\mathrm{B}} T}=0,
\end{aligned}
$$

and, for the second-order term in $p$, as

$$
\partial_{\mu}\left(n B_{q}\right)^{1-q}-\left(n B_{q}\right)^{1-q} \frac{\partial_{\mu} T}{T}=0 .
$$

Substituting eq. (15) into eq. (14), we find the relation

$$
\partial_{\mu} T=(1-q) \frac{Q}{c k_{\mathrm{B}}}\left(-\partial_{\mu} A^{v}+F^{\mu v}\right) U_{\mu} .
$$

Furthermore, let $F^{\mu v}$ be a dissymmetry tensor and make use of the relation [30]

$$
F^{\mu v}=\partial_{\mu} A^{v}-\partial_{v} A^{\mu}
$$

Eq. (16) becomes

$$
\partial_{\mu} T=(1-q) \frac{-Q}{c k_{\mathrm{B}}}\left(\partial_{\nu} A^{\mu}\right) U_{\mu} .
$$

Thus, we obtained an expression for the nonextensive parameter $q \neq 1$ for the relativistic gas under an external electromagnetic field. Eq. (18) provides a connection between the parameter $q \neq 1$ and the space-time derivatives of the temperature field of the gas as well as the four-potential, and therefore provides a clearly physical interpretation for the nonextensivity for the relativistic gas. We find that $q$ is different from unity if and only if the quantity $\partial_{\mu} T$ is different from zero. If the temperature field satisfies $\partial_{\mu} T=0$, then we have $q=1$, corresponding to the standard case in BG statistics. If the temperature field is $\partial_{\mu} T \neq 0$, then we have $q \neq 1$, corresponding to the case of NSM. Thus, the nonextensive parameter $q \neq 1$ is related closely to the spatialtemporal inhomogeneity of the temperature field of a nonequilibrium relativistic gas in an external electromagnetic field.

\section{Conclusion}

In summary, we investigated the nonextensivity and the power-law distribution of a relativistic gas under the influence of an external electromagnetic field. We derived an expression for the nonextensive parameter $q$ based on the generalized relativistic Boltzmann equation, the relativistic $q-H$ theorem, and the relativistic version of the $q$-power-law distribution function in the nonextensive $q$-kinetic theory. We thus provided the connection between the parameter $q \neq 1$ and the space-time derivatives of the temperature field of the gas as well as the four-potential, and thereby we can present a clearly physical meaning for the nonextensive parameter $q \neq 1$ for the relativistic gas. Nonextensivity is related closely to the spatial-temporal inhomogeneity of the temperature field of a nonequilibrium relativistic gas under an external electromagnetic field.

This work was supported by the "985" Program of TJU of China and the National Natural Science Foundation of China (10675088, 11175128).

1 Tsallis C. Possible generalization of Boltzmann-Gibbs statistics. J Stst Phys, 1988, 52: 479-487

2 Tsallis C. Introduction to Nonextensive Statistical Mechanics - Approaching a Complex World. New York: Springer, 2009

3 Almeida M P. Generalized entropies from first principles. Physica A, 2001, 300: 424-432

4 Du J L. The nonextensive parameter and Tsallis distribution for selfgravitating systems. Europhys Lett, 2004, 67: 893-899

5 Du J L. Nonextensivity in nonequilibrium plasma systems with Coulombian long-range interactions. Phys Lett A, 2004, 329: 262-267

6 Du J L. Hydrostatic equilibrium and Tsallis' equilibrium for self-gravitating systems. Centr Euro J Phys, 2005, 3: 376-381

$7 \mathrm{Du} \mathrm{J}$ L. What does the nonextensive parameter $q \neq 1$ stand for in self-gravitating systems? Astrophys Space Sci, 2006, 305: 247-251

8 Du J L. Nonextensivity and the power-law distributions for the systems with self-gravitating long-range interactions. Astrophys Space Sci, 2007, 312: 47-55

9 Guo L N, Du J L. The $\kappa$ parameter and $\kappa$-distribution in $\kappa$-deformed statistics for the systems in an external field. Phys Lett A, 2007, 362: 368-370 
10 Guo L N, Liu Z P, Du J L. The property of $\kappa$-parameter and $\kappa$-distribution for a relativistic gas in an electromagnetic field. Phys Lett A, 2007, 367: 431-435

11 Du J L. Erratum to: "The property of $\kappa$-deformed statistics for a relativistic gas in an electromagnetic field: $\kappa$-parameter and $\kappa$-distribution”. Phys Lett A, 2008, 372: 340-340

12 Shaikh S, Khan A, Bhatia P K. Jeans' gravitational instability of a thermally conducting, unbounded, partially ionized plasma. Z Naturforsch, 2006, 61A: 275-280

13 Shaikh S, Khan A, Bhatia P K. Jeans' gravitational instability of a thermally conducting plasma. Phys Lett A, 2008, 272: 1451-1457

14 Lavagno A, Quarati P. Metastability of electron-nuclear astrophysical plasmas: motivations, signals and conditions. Astrophys Space Sci, 2006, 305: 253-259

15 Du J L. The Chandrasekhar's condition of the equilibrium and stability for a star in the nonextensive kinetic theory. New Astron, 2006, 12: 60-63

16 Du J L. Jeans' criterion and nonextensive velocity distribution function in kinetic theory. Phys Lett A, 2004, 320: 347-351

17 Du J L. Jeans' criterion in nonextensive statistical mechanics. Physica A, 2004, 335: 107-114

18 Lima J A S, Silva R, Santos J. Plasma oscillations and nonextensive statistics. Phys Rev E, 2000, 61: 3260-3263

19 Lima J A S, Silva R, Santos J. Jeans' gravitational instability and nonextensive kinetic theory. Astron Astrophys, 2002, 396: 309-313
20 Du J L. Test of nonextensive statistical mechanics by solar sound speed. Europhys Lett, 2006, 75: 861-867

21 Liu Z P, Du J L. Dust acoustic instability driven by drifting ions and electrons in the dust plasma with Lorentzian kappa distribution. Phys Plasmas, 2009, 16: 123707

22 Liu L Y, Du J L. Ion acoustic waves in the plasma with the power-law $q$-distribution in nonextensive statistics. Physica A, 2008, 387: 4821-4827

23 Liu Z P, Liu L Y, Du J L. A nonextensive approach for the instability of current-driven ion-acoustic waves in space plasma. Phys Plasmas, 2009, 16: 072111

24 Cubero D, Dunkel J. Stationarity, ergodicity and entropy in relativistic systems. Europhys Lett, 2009, 87: 30005

25 Silva R, Lima J A S. Relativity, nonextensivity, and extended power law distributions. Phys Rev E, 2005, 72: 057101

26 Kaniadakis G. Statistical mechanics in the context of special relativity II. Phys Rev E, 2005, 72: 036108

27 Hakim R. Relativistic perfect gas in an external force field. Phys Rev, 1967, 162: 128-133

28 Hakim R. Remarks on relativistic statistical mechanics I. J Math Phys, 1967, 8: 1315-1344

29 Cercignani C, Keremer G M. The Relativistic Boltzmann Equation: Theory and Applications. Basel: Birkhauser-Verlag, 2002

30 Guo S H. Electrodynamics (in Chinese). Beijing: Higher Education Press, 1979

Open Access This article is distributed under the terms of the Creative Commons Attribution License which permits any use, distribution, and reproduction in any medium, provided the original author(s) and source are credited. 